\title{
The role of gastrostomy tube placement in advanced dementia with dysphagia: a critical review
}

This article was published in the following Dove Press journal:

Clinical Interventions in Aging

14 October 2014

Number of times this article has been viewed

\author{
Leanne S Goldberg' \\ Kenneth W Altman ${ }^{2}$ \\ 'Department of Otolaryngology, \\ Mount Sinai School of Medicine, \\ New York, NY, USA; ${ }^{2}$ Baylor College \\ of Medicine, Houston, TX, USA
}

Correspondence: Leanne S Goldberg Department of Otolaryngology, The Mount Sinai School of Medicine, 8th Floor, 5 East 98th St, New York, NY 10029, USA

Tel + I 2I2 24I I358

Email leanne.goldberg@mountsinai.org
Purpose: Over 4.5 million people in North America had a diagnosis of dementia in the year 2000, and more than half had advanced disease with potential aspiration risk. There is much controversy regarding the use and timing of enteral feeding support in these patients with dysphagia. The management of dysphagia is far more complex when considering quality of life, "comfort care" hand feeding, the use of percutaneous endoscopic gastrostomy tube (PEG), and associated mortality rates. This study seeks to critically review the literature that evaluates PEG placement in this population.

Methods: A systematic literature review of PubMed, from 1995-2012, was conducted to identify studies relating to PEG placement in dementia patients with dysphagia. The principal outcomes and related survival rates for this population were compared.

Results: In total, 100 articles were identified in the search. Of these, ten met the search criteria and were analyzed. There was one study with a $2 \mathrm{~b}$ level of evidence, one with $3 \mathrm{~b}$, and the remainder had level 4. All studies discussed long-term survival in the PEG versus non-PEG populations. No studies showed definitive evidence to suggest long-term survival rates improved in patients who underwent PEG placement as compared to those who did not. Two studies documented median survival worse in patients over age 80 with dementia and PEG placement.

Conclusion: There is presently no evidence to suggest long-term survival rates improved in patients with advanced dementia who underwent PEG placement for dysphagia. Relevance to quality of life, need for nutrition and hydration, and ethical considerations in the decision process are discussed.

Keywords: PEG, aspiration, elderly, feeding tube, swallow

\section{Introduction}

There is much controversy regarding the use and timing of enteral feeding support in patients with dysphagia and aspiration risk. The decision process becomes far more complex in those patients with advanced dementia, due to ethical and moral issues associated with the continuing comfort care per os (PO) feeds with the knowledge that the person is aspirating, and the use of percutaneous endoscopic gastrostomy tube (PEG) placement, and associated mortality rates.

Dementia is a leading cause of death in the US, with mortality affected by aspiration, hydration, and nutritional status. Data in the year 2000 show there were approximately 4.5 million people in North America with a diagnosis of dementia, and more than half progressed to the moderate to severe stages of their disease. In 2001 there were 24.3 million people in the world with dementia, and by 2040, the number is estimated to increase to over 81 million. The prevalence of dementia is estimated to double every 5 years after 65 years old, and at age 85 years, the prevalence is approximately $50 \%$. $^{1,2}$ 
Dementia is a terminal diagnosis. Alzheimer's disease and other related illnesses causing dementia are progressive, incurable, and lead to a complete loss of cognitive function, and subsequent death. A characteristic feature of the final phase of dementia, which can last from 6 months to 2 years, is loss of interest in eating, dysphagia, or both. ${ }^{3}$ An estimated $60 \%$ of nursing home residents have dementia, with approximately half $(480,000)$ being in the last stages of their disease. The prognosis and progressive clinical course affects the important decision about PEG insertion. ${ }^{4}$

Mean survival after dementia diagnosis varies between 1 and 16 years, but one-third of demented individuals live to advanced stages. ${ }^{4}$ The ability to perform activities of daily living are typically lost in a hierarchical fashion, with eating and bed mobility lost last. The most severe phase of dementia is characterized by loss of capacity to provide selfcare in basic activities of daily living, such as eating, bathing, and walking independently. In this stage there are also many behavioral symptoms that compromise quality of life for both patients and caregivers, and are sources of great stress to the latter, with institutionalization being the ultimate consequence.

Many patients and their families have limited understanding of the terminal nature of a dementia diagnosis. Due to the complexity that characterizes advanced dementia, many moral, ethical, religious, and medical decisions arise; these involve appreciating the risks, benefits, and alternatives to adjunctive enteral (PEG) feeding, "non per os" (NPO) status, and the role of "comfort care" or "compassionate PO" which is defined as continuing to feed a patient by mouth for quality of life and or comfort, in spite of and with information that the patient is aspirating and at risk for aspiration pneumonia or worse. Surprisingly, there is little discussion of these issues in the literature and in routine medical care. In this paper, we reviewed the existing literature on PEG placement in patients with dementia, in terms of subsequent mortality rates, beginning with the hypothesis that PEG does not prolong life. We also explored the impact of PEG in specific dementia groups to identify refined prognostic indicators that may be further used to develop comprehensive guidelines for the placement of PEGs in this population.

\section{Methods}

A systematic literature search was performed using PubMed, to identify studies and their levels of evidence. Key words were identified as search terms: aspiration, dysphagia, swallowing difficulty, dementia, Alzheimer, PEG, and enteral and feeding tube. The key search terms were then combined using the AND/OR operators.

Three searches gleaned the most results. The search combining dementia AND (dysphagia OR aspiration)
AND (endoscopic gastrostomy OR enteral) yielded 97 articles. The search combining (dementia OR Alzheimer) AND (dysphagia OR aspiration) AND (percutaneous endoscopic gastrostomy OR enteral OR feeding tube) yielded 99 articles, and the search gleaning the most results, 100 articles, and therefore used for this study combined the following terms (dementia OR Alzheimer) AND (dysphagia OR aspiration OR swallowing difficulty) AND (percutaneous endoscopic gastrostomy OR enteral OR feeding tube).

Our inclusion criteria required that the article 1) must have been a scientific research paper; 2) must have addressed dementia, dysphagia or aspiration risk, or PEG tube placement; and 3) must have been originally written in or translated into the English language. The exclusion criteria comprised the following: 1) studies focused solely on the pediatric population; 2) studies focused on PEG placements in other diagnoses only (studies including cohorts with dementia patients among other neurological diagnoses were not excluded if the dementia patients were separated out in the results); 3) abstracts only; and 4) nonscientific research, editorial, text book, or opinion-based papers. Once the articles were selected, the reference sections were scanned for other relevant studies. The articles were then compared on several points. First, the level of evidence for each study was determined. Second, the year of the study was noted. It was then determined whether the study was derived from or based on a systematic review of the literature. Next, we looked at the size of the study, the number of patients with dementia within the study, and specific outcomes, most importantly survival, comparing those patients with dementia who received PEGs to those who did not.

\section{Results}

The initial search yielded 100 articles. After applying the inclusion and exclusion criteria, ten articles were highlighted (the review process is summarized in the preferred reporting items for systematic reviews and meta-analyses [PRISMA] diagram as Figure 1). Overall results are summarized in Table 1, stratified by year, study design, number of subjects, level of evidence, and study findings.

\section{Retrospective studies}

The retrospective case series, which included a dementia subgroup containing 103 people with a PEG, revealed 54\% mortality at 1 month and $90 \%$ mortality at 1 year. ${ }^{1}$ A second large retrospective database spanning 10 years, which contained 8,688 demented patients who received PEGs, broke down mortality rates between males and females. ${ }^{2}$ 


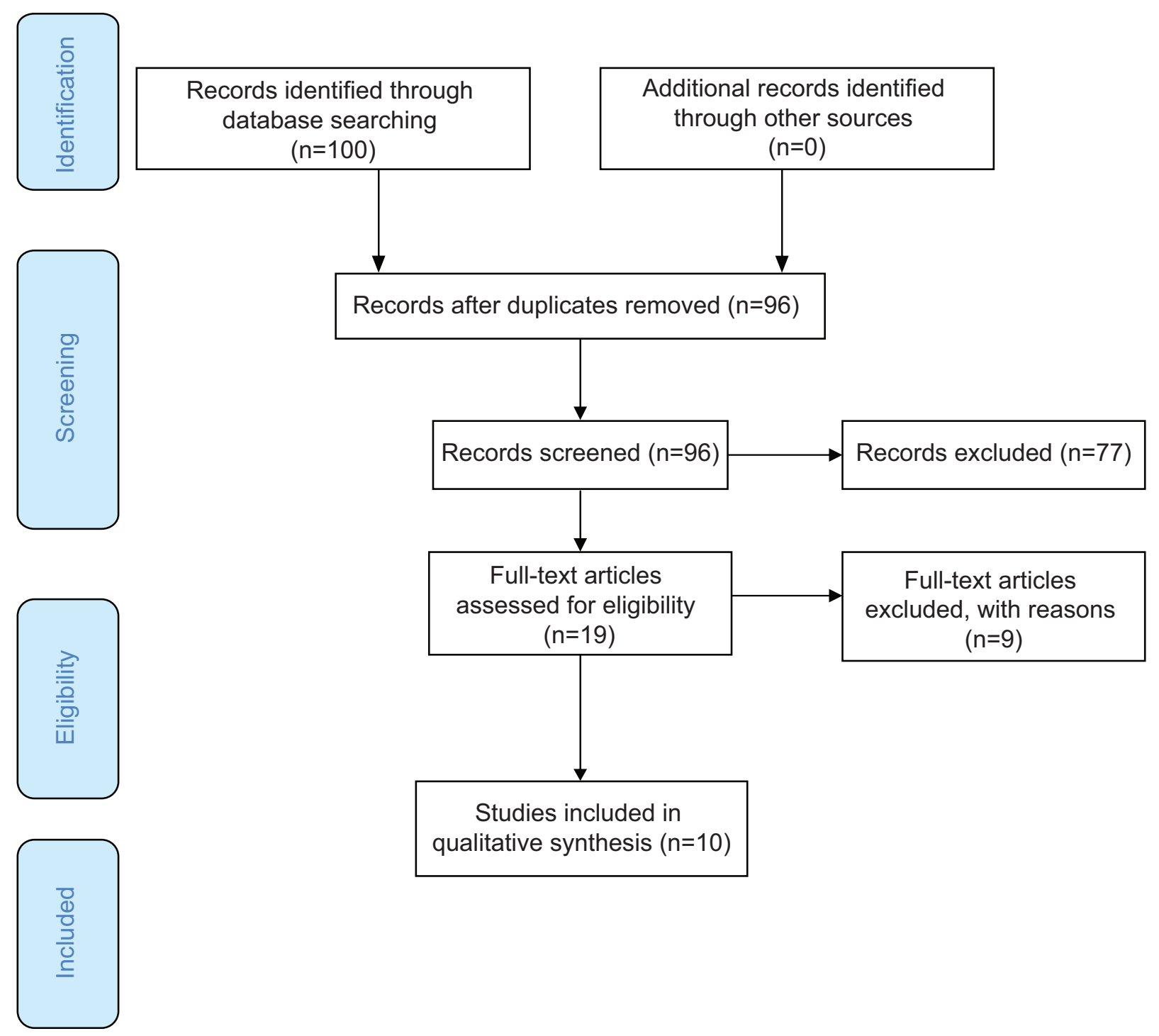

Figure I PRISMA diagram for the systematic literature search.

Abbreviation: PRISMA, preferred reporting items for systematic reviews and meta-analyses.

According to this study, at 1 year, mortality rates for males and females with dementia status post-PEG insertion were $61 \%$ and $50 \%$, respectively. At 3 years, the mortality for males and females with dementia status post-PEG was $78 \%$ and $84 \%$, respectively.

A third retrospective analysis looked specifically at indications for and survival status post-PEG in patients 65 years and older. ${ }^{3}$ The mean age of participants was 79 years old. The participants were classified into seven diagnoses: stroke; dementia; Parkinson's; other neurological diseases (mainly amyotrophic lateral sclerosis); malignancies with dysphagia; malignancies without dysphagia; and miscellaneous. The median survival for patients with dementia status post-PEG was 244 days. Mortality rates were broken down further into specific time frames. Results based on time frames were as follows: at 30 days, demented patients had 25\% mortality; at 3 months, 37\% mortality; and at 1 year, 58\% mortality.

Another retrospective study, of 90 patients with dementia and dysphagia and of average age $\sim 86$ years, revealed $14.4 \%$ mortality within 30 days and 1-year survival of $54.4 \% .{ }^{7} \mathrm{~A}$ well-known retrospective study from the VA Medical Center in Washington DC had 41 demented patients referred for PEGs. ${ }^{8}$ After educating family members, 23 received PEGs and 18 did not, secondary to family member refusal after discussion. The median survival in the group status post-PEG was 59 days compared with the group without the PEG, who survived 60 days.

A retrospective cohort study with 311 patients with PEGs, 143 with dementia, compared demented patient survival with PEG to that of patients with other diagnoses (patients without dementia). ${ }^{9}$ The 12 -month survival was $51 \%$ in 


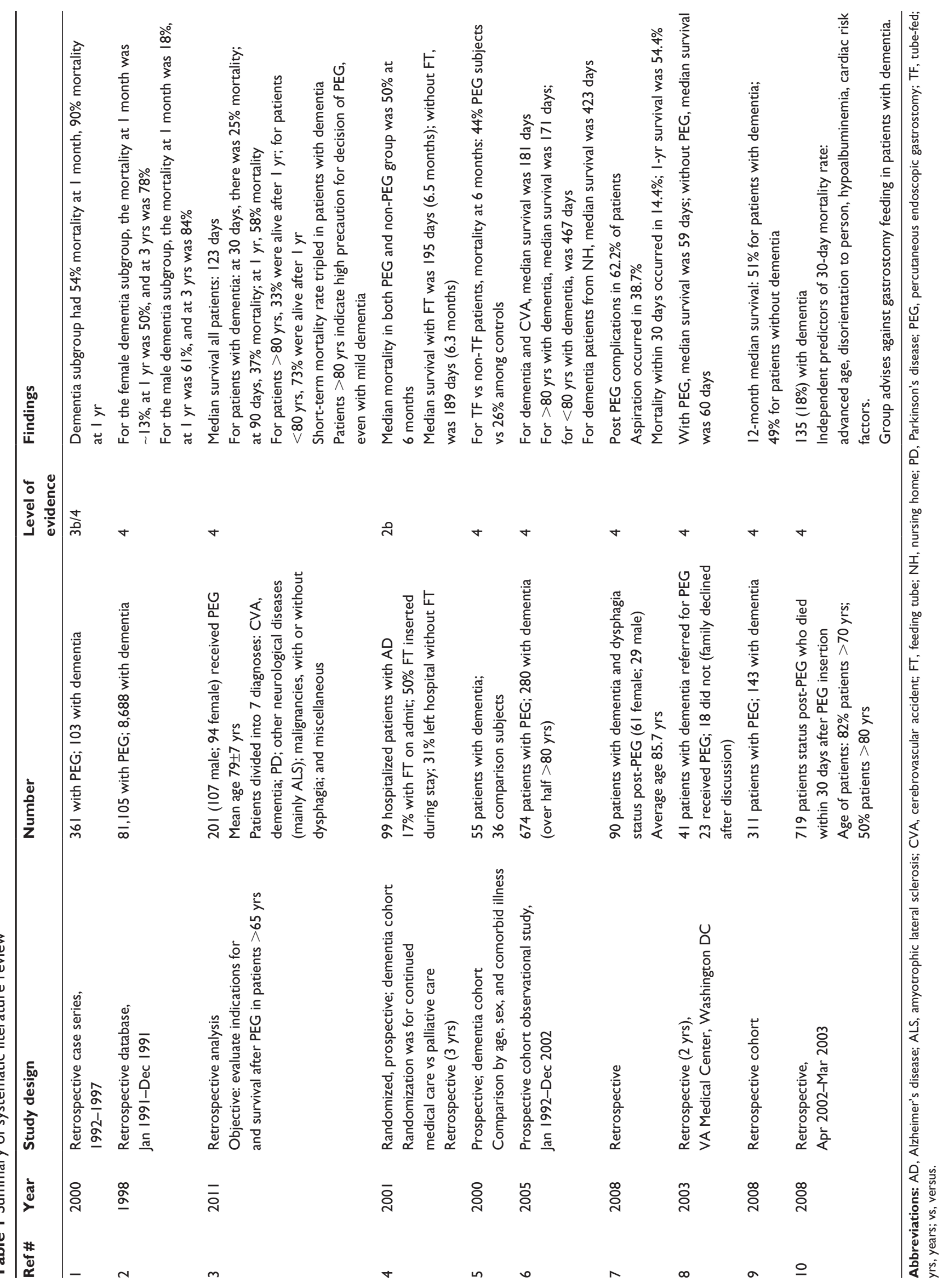


patients with dementia, and $49 \%$ in the group without dementia. More than $20 \%$ of patients with dementia lived more than 3 years after PEG. Two predictors of poor survival after PEG (among other medical issues) were patients who were male and older than 80 years of age. In this study, there was no evidence to support a poorer prognosis after PEG in elderly people with dementia compared with elderly who are cognitively intact, unless the patients are older than 80 years of age, male, or have the other poor prognostic indicators mentioned in this study, including hypoalbuminemia.

One retrospective study audited 719 patients who died within 30 days after PEG insertion. ${ }^{10}$ Of the 719 patients who received PEGs, 135 (18\%) had dementia, $82 \%$ were 70 years of age or older, and $50 \%$ were greater than 80 years of age. This study addressed the idea of careful patient selection. Of note, there was a particularly low rate $(29 \%)$ of written consent for the procedure among dementia patients. After their review, acknowledging the report that mortality rate of patients with dementia fed by gastrostomy is considerable, the group in this article advised against gastrostomy in dementia patients..$^{10}$

\section{Prospective studies}

A randomized prospective study looked at 99 hospitalized patients with advanced dementia. ${ }^{4}$ The median mortality at 6 months in the group with status "post-feeding tube" was 195 days, and in the group without the feeding tube, this was 189 days. Overall, the PEG and non-PEG median mortality at 6 months was no different, at $50 \%$. A prospective study, which included a dementia cohort of 55 patients, compared tube-fed (TF) versus (vs) non-TF patients. ${ }^{5}$ Mortality for the PEG patients at 6 months was $44 \%$ as compared with $26 \%$ among controls. The authors concluded that only $50 \%$ of demented patients with inadequate oral intake are likely to survive beyond 6 months after PEG placement and that no improvement in performance status is likely to occur in any patient. This study specifically focused on patients with inadequate oral intake secondary to dementia, in order to determine whether the nutritional parameters at the time of PEG placement would predict survival. This study recognized some benefit in performing PEG before the onset of severe hypoalbuminemia. It also recommended that prior to initiating PEG feeding, the limited benefits that would be achieved should be taken into consideration.

A prospective observational study of 674 patients included 280 dementia patients after PEG placement. ${ }^{6}$ Over half of the patients were over the age of 80 years, with an age distribution similar to that of patients undergoing
PEG placements nationally in the United States. The median survival was 171 days in patients over age 80 with dementia, which was worse when compared with other subgroups. The dementia and stroke subgroup median survival was 181 days after PEG placement. The median survival reached 423 days in demented patients from nursing homes and 467 days in demented patients younger than 80 years.

\section{Summary of results}

Results from five studies reported mortality for the patients with dementia after PEG placement. ${ }^{5-7,10,14}$ The studies reviewed reported on varied mortality time frames. Five studies revealed mortality at 30 days. The specific results were: $13 \%-18 \%,{ }^{6} 14.4 \%,{ }^{14} 18 \%,{ }^{10} 25 \%,{ }^{7}$ and $54 \% .^{5}$ One study reported 3-month mortality at $37 \% .^{7}$ Six month mortality was revealed in one study as $44 \% .{ }^{12}$ The mortality at 1 year was reported in five of the studies reviewed. Four of the five papers reported mortality approximating the $50 \%$ range. Those specific results were as follows: $45.6 \%,{ }^{14} 51 \%,{ }^{9} 50 \%-61 \%,{ }^{6}$ and $58 \%{ }^{7}$ One study reported 1 -year mortality at $90 \% .{ }^{5}$ One study revealed mortality at 3 years to be $78 \%-84 \%{ }^{6}$

Results of comparison studies regarding median mortality in TF patients vs non-TF patients were as follows. One study revealed dementia patient median survival with a feeding tube as 195 days ( 6.5 months) compared with median survival without tube feeding as 189 days (6.3 months), with no obvious difference in survival when comparing both groups. ${ }^{11}$

The following are results from individual studies. One study revealed that the group status post-PEG insertion had $44 \%$ mortality vs controls who had $26 \%$ mortality. This study revealed a higher mortality for patients with dementia who had PEGs in place. ${ }^{12}$ Another study revealed median survival after PEG to be 59 days, a low number. Yet another study revealed patients with dementia and PEG who were in nursing homes demonstrated higher median survival at 423 days, whereas dementia patients with PEG and stroke median survival was at 181 days, a similar number to the study comparing TF vs non-TF patients. ${ }^{13}$

\section{Male vs female mortality subgroup}

A large retrospective database spanning 10 years, which included 8,688 demented patients who received PEGs, broke down the mortality rates between males and females. ${ }^{2}$ This study revealed females fared slightly better, though mortality rates were still high after PEG. The female dementia subgroup had $50 \%$ mortality at 1 year and $78 \%$ at 3 years. The male dementia subgroup had $61 \%$ mortality at 1 year and $84 \%$ mortality at 3 years. 


\section{Age-based mortality subgroup}

As previously reported in retrospective studies, an analysis looked specifically at the following for patients age 65 years and older: indications for PEG placement, and survival after PEG. ${ }^{7}$ The mean age of participants was 79 years old. Patients were classified into seven diagnoses: stroke, dementia, Parkinson's, other neurological diseases (mainly amyotrophic lateral sclerosis), malignancies with or without dysphagia, and miscellaneous. Overall median survival for patients with dementia after PEG was 244 days. Mortality rates were broken down farther into specific time frames: 30 days, 3 months and 1 year.

Regarding consideration of age prior to placement of PEG in patients with dementia, only $33 \%$ of patients over age 80 were still alive after 1 year, as compared with $73 \%$ under age 80. In this particular study, dementia was not found to be a negative prognostic factor for survival in elderly patients after PEG, specifically those younger than 80 years old. Another retrospective study of 90 patients with dementia and dysphagia, whose average age was 86 years, revealed $14.4 \%$ mortality within 30 days, and 1-year survival of $54.4 \% .{ }^{14}$

\section{Discussion}

There are many controversies regarding whether or not to place a PEG in a patient with dementia who is aspirating. One involves the question of whether this intervention is sustaining life or prolonging death. Quality of life must be considered, not only for the patient but for the caregiver as well. While an in-depth discussion of these issues goes well beyond the scope of this paper, it is important to recognize some of the major problems that health care professionals, caregivers, and the patients themselves encounter.

Dementia may be classified in stages, with higher stages demonstrating more advanced disease. Stages 6 (moderatesevere dementia) and 7 (severe dementia) are listed in Table 2, with some of the hallmark signs of disease progression. Voice and speech deterioration, as well as dysphagia with aspiration risk, demonstrate the cognitive effects on laryngeal function and are included in Stage 6e. ${ }^{3}$

Discussions regarding PEG insertion do not appear to be happening during the early stages of dementia diagnosis, and many patients who receive a PEG do not have health care proxies. Patients diagnosed with dementia could be seen when presenting with initial signs of difficulty eating or swallowing, and could have regular follow-up so that aspiration risk can be assessed and potentially, intervention undertaken.

At what point are PEGs being inserted? Are they being inserted too late? Would there be a better outcome if the PEGs were inserted earlier? When is too late? Age has been shown to have an effect on survival. This is well-known and demonstrated in previous studies of PEG outcomes. ${ }^{22}$ The research has shown an increased survival rate with demented patients under age 80 , nearly double that of patients over age 80 . Sex is also a factor, coupled with age. Male sex constitutes a risk factor for higher 30-day mortality after PEG insertion.

Other considerations are: age of onset of dysphagia, age of onset of dementia, severity of dementia, and disease duration, course, and comorbidities. Every patient must still be

Table 2 Advanced stages of dementia

\begin{tabular}{|c|c|c|}
\hline Stage & Manifestations & Mean duration \\
\hline $6 \mathrm{a}$ & Ability to perform ADL becomes compromised (ie, put clothing on correctly) & 2.5 years \\
\hline $6 \mathrm{~b}$ & Lose the ability to bathe independently & 2.5 years \\
\hline $6 c$ & Lose the ability to manage the mechanics of toileting correctly & 2.5 years \\
\hline $6 d$ & Urinary incontinence & 2.5 years \\
\hline $6 e$ & $\begin{array}{l}\text { - Fecal incontinence } \\
\text { - Speech overtly breaks down in the ability to articulate. Stuttering neologisms, and/or } \\
\text { an increased paucity of speech are noted. Still able to respond to nonverbal stimuli } \\
\text { and communicate pleasure and pain via behavior }\end{array}$ & 2.5 years \\
\hline $7 a$ & $\begin{array}{l}\text { - Evident rigidity upon examination of the passive range of motion of major joints, } \\
\text { such as the elbow, in the majority of AD patients } \\
\text { - Require continuous assistance with basic ADLs for survival } \\
\text { - Speech is limited to six or fewer intelligible words }\end{array}$ & I year \\
\hline $7 \mathrm{~b}$ & $\begin{array}{l}\text { - Approximately } 40 \% \text { of } A D \text { patients manifest contractures of the elbow, wrists, } \\
\text { and fingers to the extent that they cannot move a major joint more than halfway } \\
\text { - Speech limited to a single intelligible word }\end{array}$ & 1.5 years \\
\hline $7 c$ & $\begin{array}{l}\text { - Lose the ability to ambulate independently } \\
\text { - Speech is lost }\end{array}$ & I year \\
\hline $7 d$ & Lose the ability to sit up independently & I year \\
\hline $7 e$ & Lose the ability to smile, only grimacing facial movements are observed & 1.5 years \\
\hline $7 f$ & Lose the ability to hold up their head independently & \\
\hline
\end{tabular}

Abbreviations: AD, Alzheimer's disease; ADL, activities of daily living. 
regarded as an individual. Medications should be assessed for their effects on swallowing and perhaps, medication schedules adjusted to facilitate eating, to prolong safe eating by mouth. Environmental stressors should also be addressed. Caregivers, and often health care professionals as well, do not have all the information prior to making a decision. The data should be reviewed by medical staff and family members when deciding whether and when to insert a PEG.

When the discussion and or recommendation to not insert a PEG is brought up with caregivers, this is often wrongly interpreted as a recommendation that will mean "no food" or "no care" for their loved one. This assumption results in their reluctance to take the recommendation. Caregivers need to be educated regarding quality of life PO feeding, which may be interpreted as continuing to feed patients by mouth, with the safest diet and strict aspiration precautions in place, so they may still enjoy the taste of food.

We recommend that comprehensive guidelines be established that encompass both medical and ethical issues. Prospective allocation of patient subgroups within the dementia umbrella would be optimal. Future studies that divide cohorts of retrospective studies into levels of severity of dementia would be informative. The study of the specific divisions and levels of dementia could assist in formulating future recommendations based on age, sex, nutritional status and the home environment.

Some benefits of PEG placement that were not addressed in our review include: provision of a route for reliably administering medications; supplementation of hydration and nutritional status while still allowing for "compassionate PO" or "comfort care"; as well as ensuring the bridging of nutritional needs during interval hospitalizations for conditions that may cause a transient decline in mental status. Also, while this review does not document that PEG prolongs life in this population, it is possible that a prospective study has yet to be constructed that accurately measures prolongation as well as quality of life for the patients, and their families, during their declining years.

\section{Conclusion}

There is presently no evidence in the existing literature to suggest long-term survival rates improved in patients with advanced dementia who underwent PEG placement for dysphagia and aspiration risk. However, the relevance for quality of life, need for nutrition and hydration, and ethical consideration are complex and controversial issues that warrant further study prior to achieving consensus and formalizing clinical care guidelines.

\section{Disclosure}

The authors report no conflicts of interest in this work.

\section{References}

1. Sampson EL, Candy B, Jones L. Enteral tube feeding for older people with advanced dementia. Cochrane Database of Systematic Reviews 2009;2.

2. Mitchell SL, Teno JM, Kiely DK, et al. The Clinical Course of Advanced Dementia. N Eng J Med . 2009;361:16.

3. Reisberg B, Franssen EH. Clinical Stages of Alzheimer's disease In. An Atlas of Alzheimer's Disease. de Leon MJ, editor. New York, New York: Parthenon Publishing Group; 1999:11-20.

4. Gillick M. When the Nursing home resident with advanced dementia stops eating: What is the medical director to do? J Am Med Dir Assoc. 2001;2(5):259-263.

5. Sanders DS, Carter MJ, D'Silva J, et al. Survivial analysis in PEG feeding a worse outcome in patients with dementia. Am J Gastroenterology. 2000;95(6): 1472-1475.

6. Grant MD, Rudberg M, Brody J, et al. Gastrostomy placement and mortality among hospitalized Medicare beneficiaries. JAMA. 1998;279:1973-1976.

7. Malmgren A, Hede GW, Karlstrom B, et al. Indications for Percutaneous Endoscopic Gastrostomy and survival in old adults. Food Nutr Res. 2011;55.

8. Murphy LM, Lipman TO. Percutaneous endocsopic gastrostomy does not prolong survival in patients with dementia. Arch Intern Med. 2003;163:1351-1353.

9. Higaki F, Ohishi M. Factors Predictive of Survival After Percutaneous Endoscopic Gastrostomy in the Elderly: Is Dementia Really a Risk Factor? Am J of Gastroenterol. 2008;103:1011-1016.

10. Johnston SD, Tham TC, Mason M. Death after PEG: results of the National Confidential Enquiry into Patient Outcome and Death. Gastrointestinal Endosc. 2008;68(2):223-227.

11. Meier DE, Ahronheim JC, Morris J, Baskin-Lyons S, Morrison R. High short-term mortality in hospitalized patients with advanced dementia: Lack of benefit of tube feeding. Arch Intern Med. 2001;161(4) 594-599.

12. Nair S, Hertan H, Pitchumoni C. Hypoalbuminemia is a poor predictor of survival after PEG in elderly patients with dementia. Am J Gastroenterol. 2000;95(1):133-136.

13. Rimon E, Nadya K, Shmuel L. Percutaneous Eendoscopic Gastrostomy; evidence of different prognosis in various patient subgroups. Age and Ageing. 2005;34:353-357.

\section{Dovepress}

\section{Publish your work in this journal}

Clinical Interventions in Aging is an international, peer-reviewed journal focusing on evidence-based reports on the value or lack thereof of treatments intended to prevent or delay the onset of maladaptive correlates of aging in human beings. This journal is indexed on PubMed Central, MedLine,
CAS, Scopus and the Elsevier Bibliographic databases. The manuscript management system is completely online and includes a very quick and fair peer-review system, which is all easy to use. Visit http://www.dovepress. $\mathrm{com} /$ testimonials.php to read real quotes from published authors. 